\title{
Biogene Calcitfällung im Bodensee - Prozessverständnis und Modellierung
}

Die biogene Calcitfällung ist ein in vielen Hartwasserseen beobachtetes Phänomen während starken Algenwachstums („Algenblüte“). Im Rahmen des Verbundprojektes BodenseeOnline wurde dieser für den Bodensee primäre anorganische Partikelbildungsprozess weiterführend untersucht. Durch die Kombination von Laborversuchen unter bodenseespezifischen Randbedingungen und einem Gewässermonitoring konnte ein Partikelwachstumsmodell entwickelt werden, mit dem es möglich ist, biogen induzierte Calcitfällungsereignisse im Bodensee zu identifizieren.

\section{Einleitung}

Limnische Ökosysteme werden durch eine Vielzahl miteinander verknüpfter chemischer, physikalischer, biologischer und geologischer Prozesse beeinflusst. Eine Schlüsselstellung in der Verbindung zwischen Biospäre und Hydrosphäre besitzt dabei der Kohlenstoffkreislauf. Neben der Bedeutung als Hauptbestandteil der organischen Biomasse steht der Kohlenstoff vor allem auch im Zentrum anorganischer partikelbildender Prozesse [1]. Im Bodensee ist die biogene Calcitfällung der primäre anorganische Partikelbildungsprozess. Die Induzierung eines solchen Ereignisses erfolgt durch die 2 bis 3 Mal jährlich auftretenden Algenblüten. Die stark ansteigende photosynthetische Aktivität bedingt einen $\mathrm{pH}$-Anstieg, eine Verschiebung im Carbonatgleichgewicht und führt folglich zur Bildung von Calcitkristallen. Im Rahmen von BodenseeOnline wurde die biogen induzierte Bildung von Calcit auf der Basis bestehender Untersuchungen [2], [3] und verbesserter Untersuchungsmethoden weiterführend untersucht, wobei Fällungsexperimente im Labormaßstab und Freilandbeobachtungen die Grundlage der Prozessaufklärung darstellten. Ziel der Untersuchungen war es, ein Partikelwachstumsmodell zu entwickeln, um Aussagen darüber treffen zu können, unter welchen Umweltbedingungen es zu einem Calcitfällungsereignis kommt. Hierzu wurden unter kontrollierten Bedingungen Fällungsexperimente im Labor durchgeführt sowie ein Monitoringprogramm der wichtigsten
Parameter für die Calcitfällung im Bodensee etabliert.

\section{Fällungsexperimente im Labormaßstab}

Für die Aufklärung der Induzierungsund Wachstumsprozesse von Calcitkristallen wurden Laborversuche unter Bodensee-spezifischen Randbedingungen durchgeführt. Hierfür wurde ein Reaktorsystem entwickelt, mit dem es möglich war, alle wichtigen Parameter ( $\mathrm{pH}-$ Wert, elektr. Leitfähigkeit, Sauerstoff etc.) der Calcitbildung mit einer sehr hohen zeitlichen Auflösung zu erfassen [4]. Eine weitere Anfor- derung bestand darin, dass Fällungsereignisse sowohl anorganisch $(\mathrm{NaOH}-\mathrm{Zu}$ gabe) als auch biogen (Verwendung von Algen) induziert werden können. Für die Versuchsreihen mit im Bodensee ansässigen Algenarten wurden Lichtbänke installiert. Dadurch konnten Tag-NachtZyklen simuliert werden, die auf den natürlichen Gegebenheiten im Bodensee basierten [5]. Neben der Erfassung der thermodynamisch relevanten Parameter ist die Partikelkonzentration eine notwendige Eingangsgröße für die mathematische Beschreibung der Kristallwachstumskinetik. Hierfür wurde in das Reaktorsystem eine kontinuierliche Partikelanalyse integriert. Zusätzlich bestand die

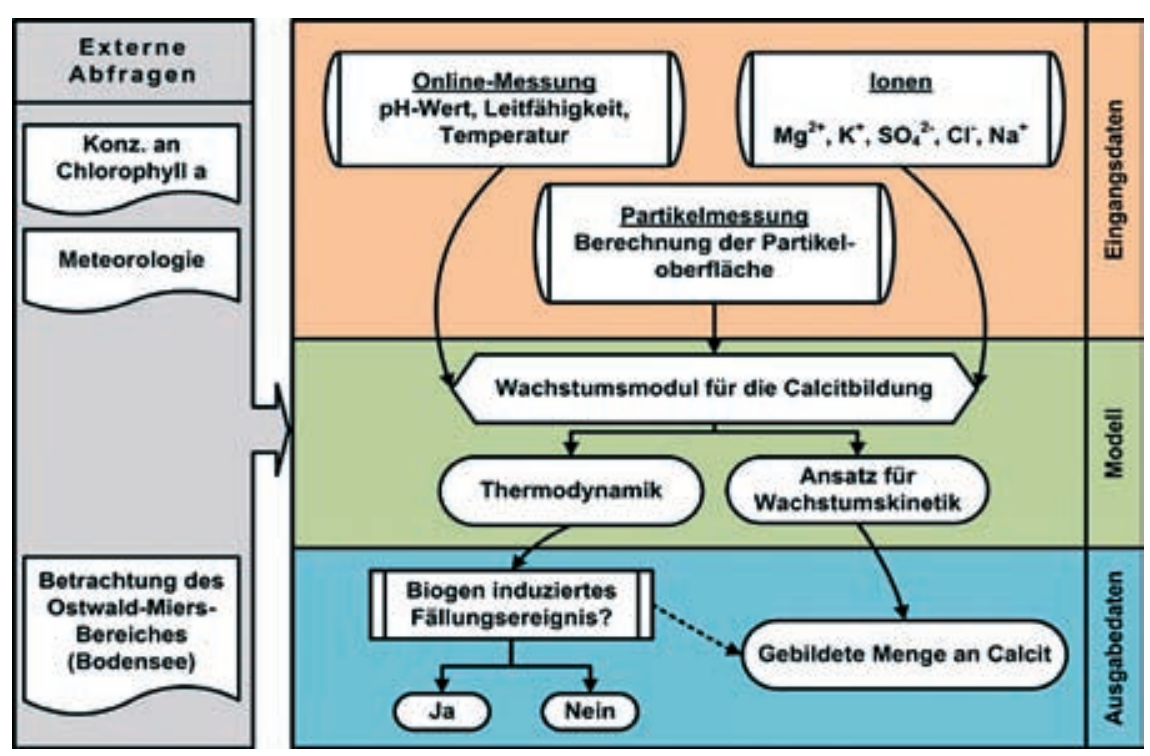

Bild 1: Schematische Darstellung des Identifizierungs- und Berechnungsmoduls für die biogene Calcitfällung 
Möglichkeit, Proben aus dem Reaktor für die Bestimmung weiterer chemischer Messgrößen und zur mikroskopischen Bildanalyse zu entnehmen. Mit Hilfe dieses Versuchaufbaus war es möglich, den Prozess der Calcitkristallbildung detailliert zu studieren. Dies gilt sowohl für die Betrachtung von Kristallwachstumsprozessen als auch die Untersuchung verschiedener im Bodensee ansässiger Algenspezies hinsichtlich ihrer Fähigkeit, ein Calcitfällungsereignis zu induzieren.

\section{Das Partikelwachstumsmodell}

Auf Basis der theoretischen Grundlagen der Calcitkristallbildung wurde ein Wachstumsmodell entwickelt. Hierbei wurde die zugrunde liegende Thermodynamik [6] mit kinetischen Wachstumsansätzen gekoppelt [7], [8], [9], [10]. Somit bestand die Möglichkeit, die pro Zeiteinheit gebildete Menge an Calcit zu berechnen. Dies fand sowohl Anwendung in den Versuchsreihen im Labormaßstab als auch in weiterführenden Auswertungen des während des Gewässermonitorings erfassten Datensatzes. Im Rahmen des Informationssystems BodenseeOnline bestand eine weitere Anforderung darin, Phasen biogener Calcitbildung anhand der zur Verfügung stehenden Daten identifizieren zu können. In Bild 1 ist schematisch die Vorgehens- weise dargestellt. So wird nach Eingang aller notwendigen Messdaten und unter Berücksichtigung weiterer externer Randparameter (Meteorologie etc.) der thermodynamische Zustand berechnet. Anhand von Leitparametern für die Calcitausfällung (z. B. Übersättigung bezüglich $\mathrm{CaCO}_{3}$ ) können Aussagen über das mögliche Vorhandensein eines Fällungsereignisses im Bodensee formuliert werden. Weiterführend kann bei ausreichender Datenlage die Menge des gebildeten Calcits lokal berechnet werden.

\section{Ausgewählte Ergebnisse des Gewässermonitorings}

Zur Erfassung von biogenen Calcitfällungsereignissen im Bodensee ist zwischen März und September 2007 ein Gewässermonitoring durchgeführt worden. Hierzu wurden im Rahmen von wöchentlichen Seeausfahrten (Messboje Uni Kons$\operatorname{tanz}$ ) sowohl der limnologische Zustand als auch die wichtigsten chemisch/physikalischen Wassergüteparameter in Form von Vertikalprofilen erfasst. Insgesamt kam es im Untersuchungszeitraum zu drei Phasen biogen induzierter Calcitfällungsereignisse. Das Bild 2 zeigt, dass bereits Mitte April ein signifikanter $\mathrm{pH}-$ Wertanstieg im Epilimnion gemessen wurde, der mit einer sehr hohen Wahrscheinlichkeit

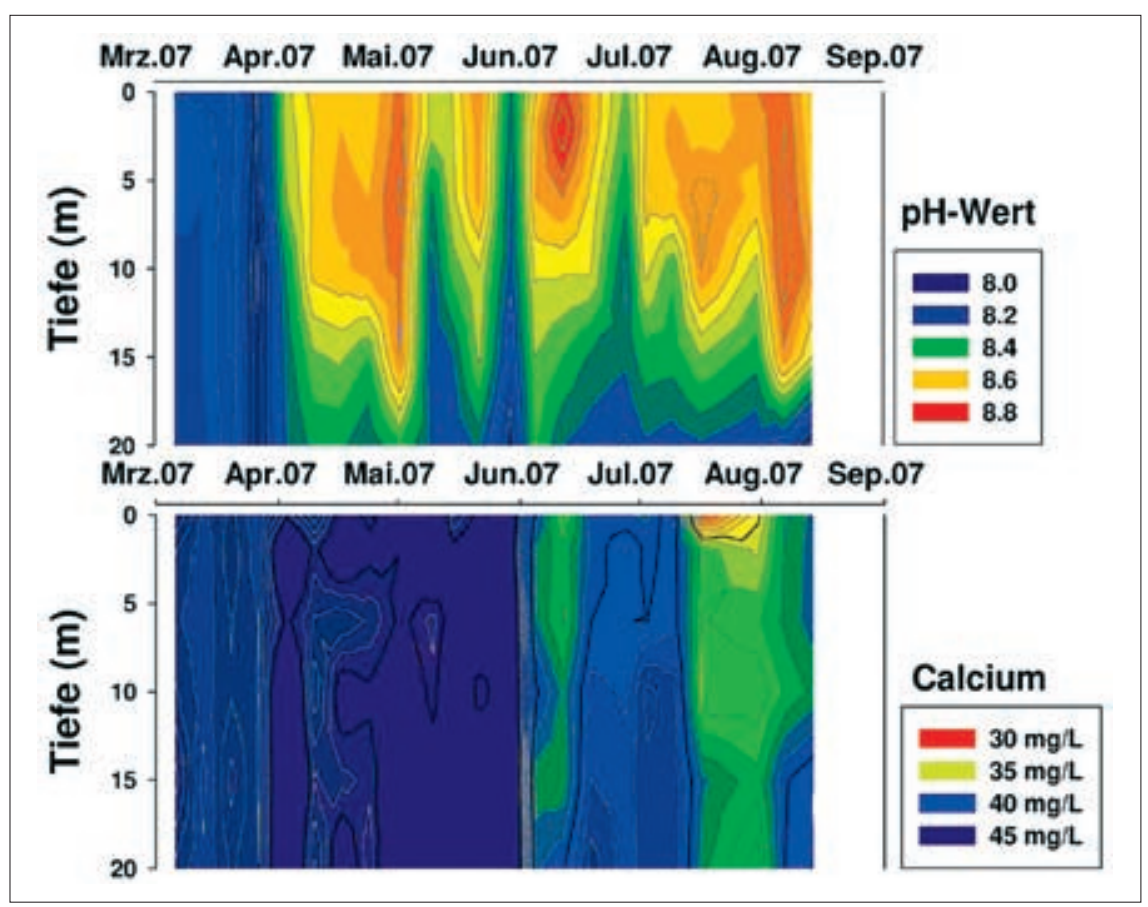

Bild 2: Darstellung der Vertikalprofilmessung für den pH-Wert und für die Konzentration des gelösten Calciums im Untersuchungszeitraum März bis Mitte August 2007

aus einer ersten Algenblüte resultierte. Mitte Juni und Anfang August sind deutliche Calcitfällungsphasen erfasst worden. Neben den erhöhten pH-Werten und gestiegenen Konzentrationen an Chlorophyll a (hier nicht dargestellt), belegten die Konzentrationsabnahmen an gelöstem Calcium die Ausfällung von Calcit (Bild 2). Die Bestimmung der Konzentration an gelöstem Calcium ergab, dass Anfang August die am stärksten ausgeprägte Bildung von Calcit erfasst wurde, wobei die $\mathrm{Ca}^{2+}$ Konzentration, gemessen am Jahresmittelwert, um ca. 33 \% abnahm. Parallel gemessene Parameter, wie z. B. die Partikelkonzentration, bestätigten das Auftreten dieser drei Calcitfällungsereignisse.

\section{Zusammenfassung}

Durch die Kombination von Laborexperimenten sowie Freilandbeobachtung und den daraus gewonnen Erkenntnissen wurde ein Partikelwachstumsmodell erstellt, mit dem es möglich ist, Phasen von biogener Calcitfällung im Bodensee zu identifizieren. So konnte mithilfe des entwickelten Reaktorsystems der Vorgang der Calcitkristallbildung eingehend untersucht werden. Hierzu zählten die Betrachtung der Wachstumsphasen von Calcitkristallen und die Aufklärung der Fähigkeit von im Bodensee vorkommenden Algenspezies, ein Calcitfällungsereignis zu induzieren. Im Rahmen des Gewässermonitorings 2007 sind insgesamt 3 Phasen biogener Calcitausfällungen beobachtet worden. Durch den erhobenen Datensatz können Aussagen über das zeitliche Einsetzen, das Ausmaß und die vertikale Ausdehnung getroffen werden. Die Möglichkeit, diesen für den Bodensee primären anorganischen Partikelbildungsprozess $\mathrm{zu}$ identifizieren und qualitativ/quantitativ beschreiben zu können, vereinfacht nicht zuletzt den am Bodensee befindlichen Wasserwerken die Überwachung des für die Trinkwasserversorgung verwendeten Bodenseewassers.

\section{Danksagung}

Die hier vorgestellte Arbeit entstand im Rahmen des vom Bundesministerium für Bildung und Forschung geförderten Forschungsprojektes mit dem Förderkennzeichen 02WT00552, das Teil des Verbundforschungsprojektes BodenseeOnline ist. Die Autoren bedanken sich für die zuteil gewordene finanzielle Unterstürzung. 
Sebastian Kempke, Roland Schick, Karsten Rinke and Karl-Otto Rothhaupt

\section{Calcite Precipitation in Lake Constance - Understanding and Modelling}

Calcite precipitation is a phenomenon frequently observed in many hard water lakes during an immense growth of phytoplankton ("algal bloom"). This primary inorganic process of particle formation was intensively studied, using Lake Constance as an example. In laboratory experiments the mechanism of organically and inorganically induced calcite precipitation was quantitatively analyzed. Based on thermodynamic and kinetic laws we characterized the crystal growth rate $\mathrm{R}$ in precipitation experiments under different physical, biological and chemical boundary conditions. Within a field campaign in 2007 three phases of calcite precipitation were detected. The combination of experiments and field campaigns enabled us to build a crystal growth model for predicting calcite precipitations by using online measuring methods ( $\mathrm{pH}$-value, conductivity, temperature etc.).

\section{Autoren \\ Sebastian Kempke \\ Dr.-Ing. Roland Schick}

Zweckverband Bodensee-Wasserversorgung

Betriebs- und Forschungslabor

Süßenmühle 1, 78354 Sipplingen

Sebastian.Kempke@zvbwv.de

Roland.Schick@zvbwv.de

\section{Dr. Karsten Rinke}

Prof. Dr. Karl-Otto Rothhaupt

Universität Konstanz

Limnologisches Institut
Mainaustr. 252

78464 Konstanz

Karsten.Rinke@uni-konstanz.de

Karl.Rothhaupt@uni-konstanz.de

\section{Literatur}

[1] Sigg, L.: Aquatische Chemie. 4. Aufl.; Zürich: Vdf Hochschulverlag, 1996.

[2] Stabel, H.-H.: Calcite precipitation in Lake Constance: chemical equilibrium, sedimentation, and nucleation by algae. In: Limnol. Oceanogr. 31 (1986), S. 1 081-1 093.
[3] Kleiner, J.: Untersuchungen zur Calcitausfällung im Bodensee. In: Konstanz: HartungGorre Verlag, Konstanzer Dissertationen (1991), Band 306.

[4] Kempke, S.; Schick, R.; Rinke, R.; Rothhaupt K.-O.: Zeitlich hochaufgelöste Untersuchung der biogen induzierten Calcitbildung. In: Tagungsbericht der Deutschen Gesellschaft für Limnologie (DGL), Dresden, 2006.

[5] Hoffmann, H.; Lorke, A.; Peeters, F.: Wave-induced variability of the underwater light climate in the litoral zone. In: Proceedings of the International Association of Theoretical and Applied Limnology, Volume 30.

[6] Norm DIN 38404 Teil C 10: Calcitsättigung eines Wassers. Berlin: Beuth Verlag GmbH, 1995.

[7] Johannsen K.: Mechanismen der Entcarbonisierung bei der Aufbereitung von Trinkwasser. In: . Veröffentlichungen aus dem Technologiezentrum Wasser, Karlsruhe, zugl.: Hamburg-Harburg, Technische Universität, Habil.Schrift, 2000.

[8] Johannsen K.: Effect of Iron and Manganese lons on the Crystal Growth of Calcium Carbonate. In: Acta hydrochim. hydrobiol. 25 (1997), Nr. 4, S. 202-207.

[9] Reddy M. M.; Nancollas, G. H.: The crystallization of calcium carbonate: I. Isotopic exchange and kinetics. In: J. Colloid Interf. Sci. (1971), Nr. 36, S. 166-172.

[10] Reddy M. M.; Nancollas, G. H.: The crystallization of calcium carbonate. II. Calcite growth mechanism. In: J. Colloid Interface Sci. 4 (1971), Nr. 37, S. 824-830. 